\title{
ELECTRONIC STRUCTURE OF LIQUID TRANSITION AND RARE-EARTH METALS AND THEIR ALLOYS (*)
}

\author{
G. BUSCH, H.-J. GUNTHERODT, H. U. KÜNZI, \\ H. A. MEIER and L. SCHLAPBACH \\ Laboratorium für Festkörperphysik, ETH Zürich, \\ Switzerland \\ and J. KELLER \\ Facultad de Quimica, University of Mexico, Mexico
}

\begin{abstract}
Résumé. - Les récents progrès expérimentaux concernant les métaux de transition, les métaux des terres rares et leurs alliages liquides sont passés en revue. Les résultats sont comparés avec les théories actuelles qui tiennent compte des états $3 \mathrm{~d}$, $4 \mathrm{f}$ et $5 \mathrm{~d}$ respectivement.
\end{abstract}

\begin{abstract}
The recent progress in the experimental work on liquid transition and rare earth metals and their alloys is reviewed. The results are compared with recent theories taking into account the $3 \mathrm{~d}, 4 \mathrm{f}$ and $5 \mathrm{~d}$ states, respectively.
\end{abstract}

1. Introduction. - The data on the Hall coefficient, the Lorenz number, the electrical and thermal resistivity, the thermopower and the magnetic susceptibility of liquid normal metals and their alloys can be interpreted in terms of the nearly-free-electron model [1]. This model may not be valid in liquid transition and rare-earth metals and their alloys due to the presence of the $3 \mathrm{~d}, 4 \mathrm{f}$ and $5 \mathrm{~d}$ states.

The main object of this paper is to review the measurements of the Hall coefficient, the electrical resistivity and the magnetic susceptibility of the liquid transition metals $\mathrm{Mn}, \mathrm{Fe}, \mathrm{Co}, \mathrm{Ni}$ and the light rare-earth metals $\mathrm{La}, \mathrm{Ce}, \mathrm{Pr}, \mathrm{Na}$ and their concentrated alloys with liquid normal metals. Simple explanations are given for the observed behavior.

New results of the electrical resistivity of liquid La, $\mathrm{Gd}, \mathrm{Tb}$ and $\mathrm{Eu}$, of the Hall coefficient and the electrical resistivity of liquid $\mathrm{Co}-\mathrm{Ce}$ and of the magnetic susceptibility of liquid alloys of $\mathrm{Ce}$ with $\mathrm{Cu}, \mathrm{Sn}, \mathrm{La}$ and $\mathrm{Pr}$ and of liquid Co-La and Co-Pr are presented.

2. Liquid transition metals and their alloys. 2.1 HALL COEFFICIENT. - A survey of the Hall coefficients of liquid transition metals and their alloys with $\mathrm{Ge}$ is given in figure 1 . From the data it follows that only $\mathrm{Ni}$ has a negative Hall coefficient whereas $\mathrm{Mn}, \mathrm{Fe}$ and $\mathrm{Co}$ have positive Hall coefficients. The Hall coefficients of liquid Ge-Ni alloys are always negative and the qualitative behavior does not differ very much from the $\mathrm{Cu}-\mathrm{Ge}$ alloys. The Hall coefficients of liquid $\mathrm{Cu}-\mathrm{Ge}$ are in agreement

(*) Dedicated to dipl. phys. ETH Annamarie Zimmermann, who died 12th of August, 1973.

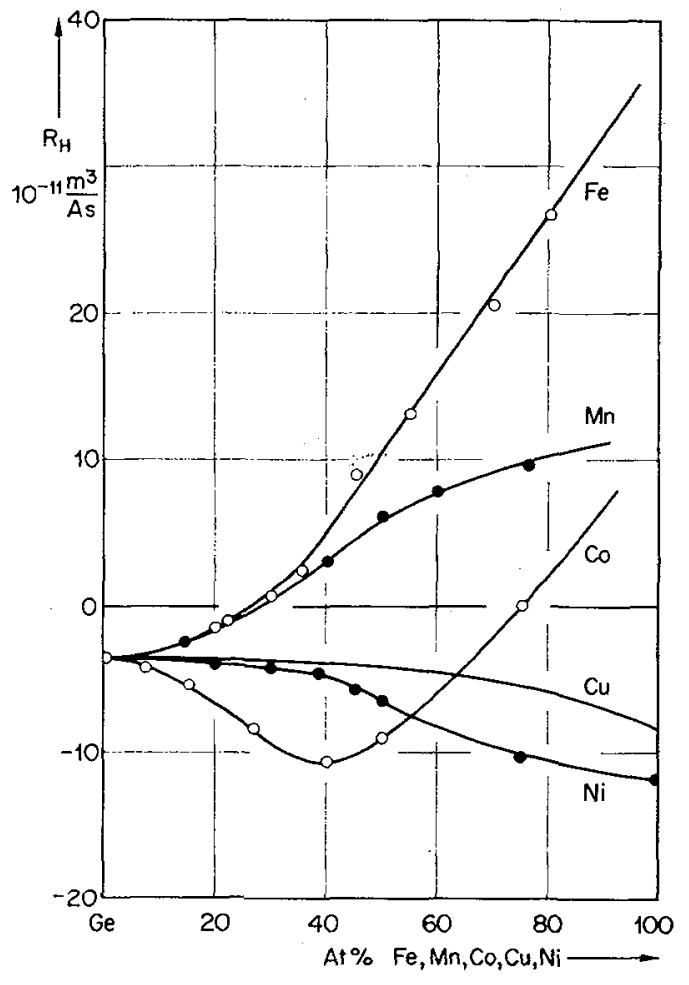

FIG. 1. - The Hall coefficients of liquid alloys of Ge with transition metals.

with the free-electron model. The number of conduction electrons varies between one for $\mathrm{Cu}$ and four for $\mathrm{Ge}$ in these alloys. The behavior of the Ge-alloys with $\mathrm{Mn}, \mathrm{Fe}$ and $\mathrm{Co}$ is characterised by a change of 
sign of the Hall coefficient and positive Hall coefficients for the transition metal rich side.

So far there have been several attempts to explain positive Hall coefficients, but it is still an open question, however, what model might be best [1].

2.2 EleCtrical RESISTIVITY. - Figure 2 shows the electrical resistivity of liquid $\mathrm{Mn}, \mathrm{Fe}, \mathrm{Co}, \mathrm{Ni}$ and $\mathrm{Cu}$ at their melting points as a function of the position in the periodic table. The electrical resistivity of liquid $\mathrm{Mn}, \mathrm{Fe}$, $\mathrm{Co}$ and $\mathrm{Ni}$ decreases with increasing group number in the periodic table.

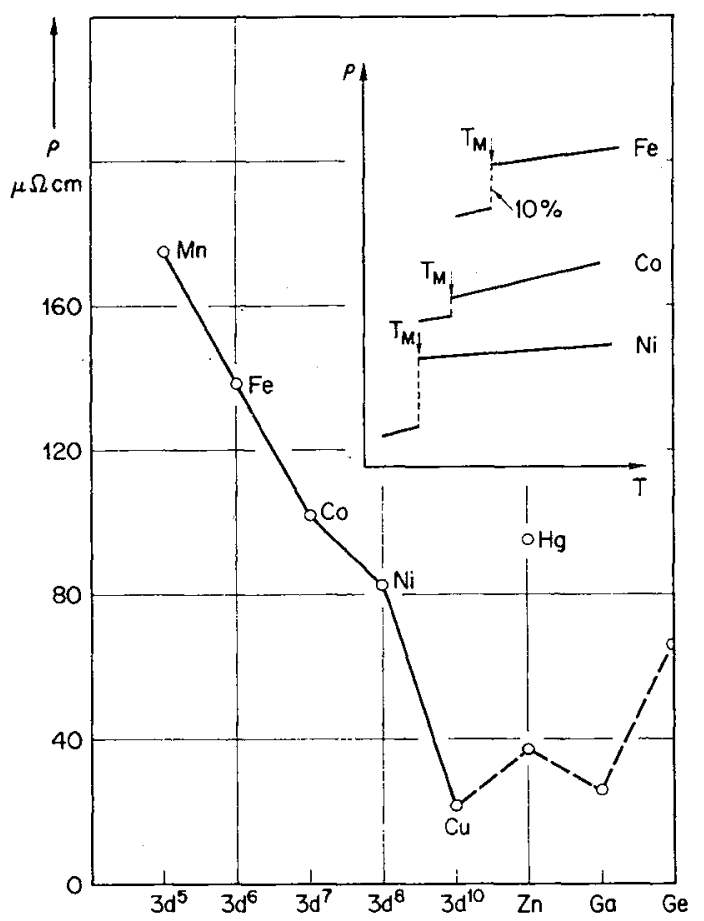

FIG. 2. - The electrical resistivity of liquid transition metals at their melting points.

These results can be interpreted by the theory of the electrical resistivity for liquid transition metals suggested by Evans et al. [2]. The electrical resistivity is given by

$$
\rho=\frac{3 \pi \Omega}{4 e^{2} \hbar v_{\mathrm{F}}^{22} k_{\mathrm{F}}^{4}} \int_{0}^{2 k_{\mathrm{F}}} a(K)|t(K)|^{2} K^{3} \mathrm{~d} K
$$

where $\Omega$ is the atomic volume, $v_{\mathrm{F}}$ the Fermi velocity, $t(K)$ the single-site $t$-matrix and $a(K)$ the structure factor.

If the d-phase shift $\eta_{2}$ is dominant at the Fermi energy in transition metals the electrical resistivity can be written in the following approximation :

$$
\rho=\frac{30 \pi^{2} \hbar^{3}}{m e^{2} k_{\mathrm{F}}^{22} E_{\mathrm{F}} \Omega} a\left(2 k_{\mathrm{F}}\right) \frac{\Gamma^{2}}{\Gamma^{2}+4\left(E_{\mathrm{ros}}-E_{\mathrm{F}}\right)^{2}}
$$

where $\Gamma$ is the width and $E_{\text {res }}$ is the position of the scattering resonance, approximately the center of the 3d-band.
There are two main contributions to the electrical resistivity of liquid transition metals : pair correlation function and resonance scattering. The liquid transition metals have the same pair correlation function as liquid $\mathrm{Cu}$. Therefore, the contribution arising from the correlation function will be nearly the same and will be small if we assume only one conduction electron. The resonance scattering decreases from $\mathrm{Mn}$ to $\mathrm{Ni}$ due to the increasing difference between the resonance energies of the 3d-states and the Fermi energy in these transition metals. $E_{\text {res }}$ and $E_{\mathrm{F}}$ are close together in $\mathrm{Mn}$ with an approximately half filled $3 \mathrm{~d}$-band.

The numerical calculations of the electrical resistivity require accurate values for the potential, the phase shifts $\eta_{2}$, the Fermi energy $E_{F}$ and the Fermi wave number $k_{\mathrm{F}}$.

Figure 3 shows the typical behavior of the electrical resistivity and its temperature coefficient as a function of concentration for two examples of liquid alloy types. The Au-Ni alloys show nearly the same behavior as the $\mathrm{Au}-\mathrm{Ag}$ group of liquid normal metals. The resistivity has the same concentration dependence as predicted by the Nordheim rule for the solid state and positive temperature coefficients are observed over the whole concentration range.

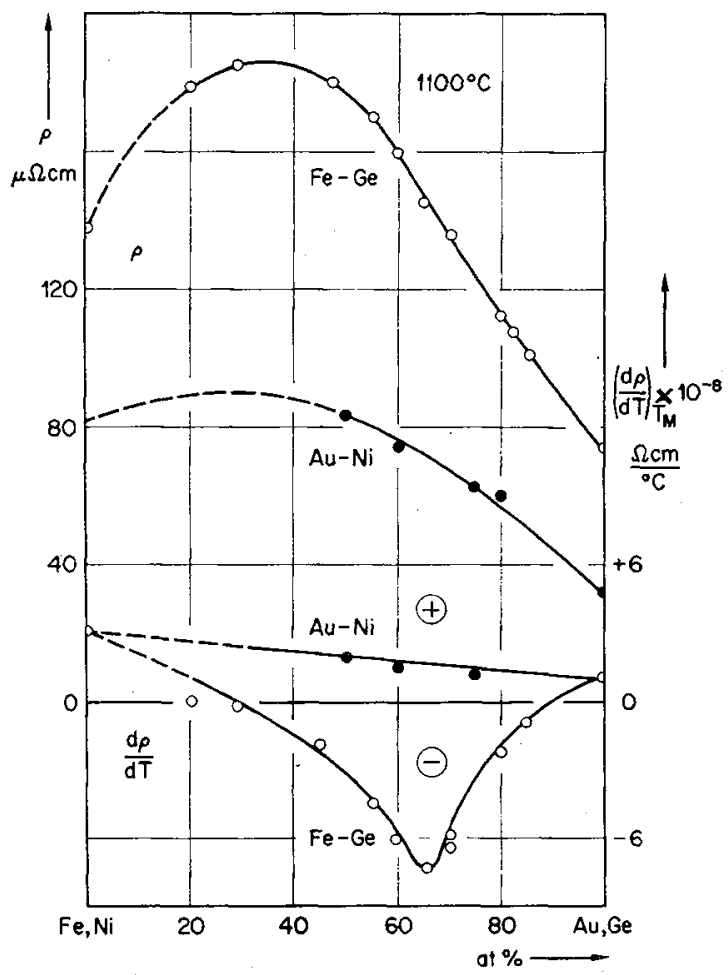

FIG. 3. - The electrical resistivity and its temperature coefficient for $\mathrm{Fe}-\mathrm{Ge}$ and $\mathrm{Au}-\mathrm{Ni}$ alloys.

The Fe-Ge alloys show a large maximum in the electrical resistivity as a function of concentration and negative temperature coefficients of the electrical resistivity. 
An extension [3, 4] of the Faber-Ziman formula to liquid alloys of transition metals offers an explanation for the observed resistivity data. The electrical resistivity is given by

$$
\rho=\frac{3 \pi \Omega}{4 e^{2} v_{\mathrm{F}}^{2} \hbar k_{\mathrm{F}}^{4}} \cdot \int_{0}^{2 k_{\mathrm{F}}}|U(K)|^{2} K^{3} \mathrm{~d} K
$$

with

$$
\begin{aligned}
|U(K)|^{2}=c_{\mathrm{A}} \mid & \left.t_{\mathrm{A}}\right|^{2}\left(1-c_{\mathrm{A}}+c_{\mathrm{A}} a_{\mathrm{AA}}\right)+ \\
& +c_{\mathrm{B}}\left|t_{\mathrm{B}}\right|^{2}\left(1-c_{\mathrm{B}}+c_{\mathrm{B}} a_{\mathrm{BB}}\right) \\
& +c_{\mathrm{A}} c_{\mathrm{B}}\left(t_{\mathrm{A}}^{*} t_{\mathrm{B}}+t_{\mathrm{A}} t_{\mathrm{B}}^{*}\right)\left(a_{\mathrm{AB}}-1\right)
\end{aligned}
$$

where $c_{\mathrm{A}, \mathrm{B}}$ are the concentrations, $t_{\mathrm{A}, \mathrm{B}}$ the $t$-matrices of the components $\mathrm{A}$ and $\mathrm{B}$, and $a_{\mathrm{AA}}, a_{\mathrm{BB}}$ and $a_{\mathrm{AB}}$ the partial structure factors of the alloy.

In order to discuss binary alloys of transition metals from a fundamental point of view, the three partial structure factors are required. These quantities are not yet known for alloys of transition metals. A qualitative discussion has been based on empirical data [5] of the structure, and numerical calculations have been carried out by using the hard sphere model results of the Percus-Yevick equation.

The important difference between the two groups of $\mathrm{Au}-\mathrm{Ni}$ and Co-Ge alloys is the position of $2 k_{\mathrm{F}}$ for the pure monovalent noble metal or the polyvalent normal metal compared with the $K_{\mathrm{p}}$-value of the first peak of $a(K)$ of the pure transition metals. The $K_{\mathrm{p}}$-value of the transition metal is assumed to be representative of the $K_{\mathrm{p}}$ value of $a_{\mathrm{AB}}(K)$ of the alloy. Then we have the relation $2 k_{\mathrm{F}}(\mathrm{Au})<K_{\mathrm{p}}$ (alloy) $<2 k_{\mathrm{F}}(\mathrm{Ge})$. In the Au-Ni group the $2 k_{\mathrm{F}}$ values are always smaller than the $K_{\mathrm{p}}$ value of the alloys. In the Co-Ge group the small $2 k_{\mathrm{F}}$ values of the pure transition metals increase and move through the $K_{\mathrm{p}}$ value of the alloy on alloying with polyvalent metals. Therefore, the resistivity increases since the contribution of the structure factor increases. Furthermore, negative temperature coefficients occur.

The results of numerical calculations [4] of the concentration dependence of the electrical resistivity of the alloy systems $\mathrm{Ni}-\mathrm{Au}, \mathrm{Fe}-\mathrm{Au}, \mathrm{Ni}-\mathrm{Sn}$ and $\mathrm{Fe}-\mathrm{Ge}$ are in reasonable agreement with the experiment.

2.3 MAGNeTIC SUSCEPTIBILITY. - The reciprocal susceptibility of liquid $\mathrm{Ni}, \mathrm{Mn}, \mathrm{Fe}$ and $\mathrm{Co}[6]$ as a function of temperature is shown in figure $4 a$. The temperature coefficient of the reciprocal susceptibility of $\mathrm{Fe}, \mathrm{Co}$ and $\mathrm{Ni}$ is positive. Only pure Mn shows an increase of the susceptibility with increasing temperature above the melting point. The changes of the magnetic susceptibility at the melting point are small for $\mathrm{Mn}, \mathrm{Co}$ and $\mathrm{Ni}$. Figure $4 b$ shows the values of the susceptibility at the melting points. They increase from $\mathrm{Mn}$ to $\mathrm{Fe}$, reach a maximum at $\mathrm{Co}$ and decrease to $\mathrm{Ni}$ and $\mathrm{Cu}$. For comparison the PauliLandau susceptibility of some liquid normal metals is also shown.

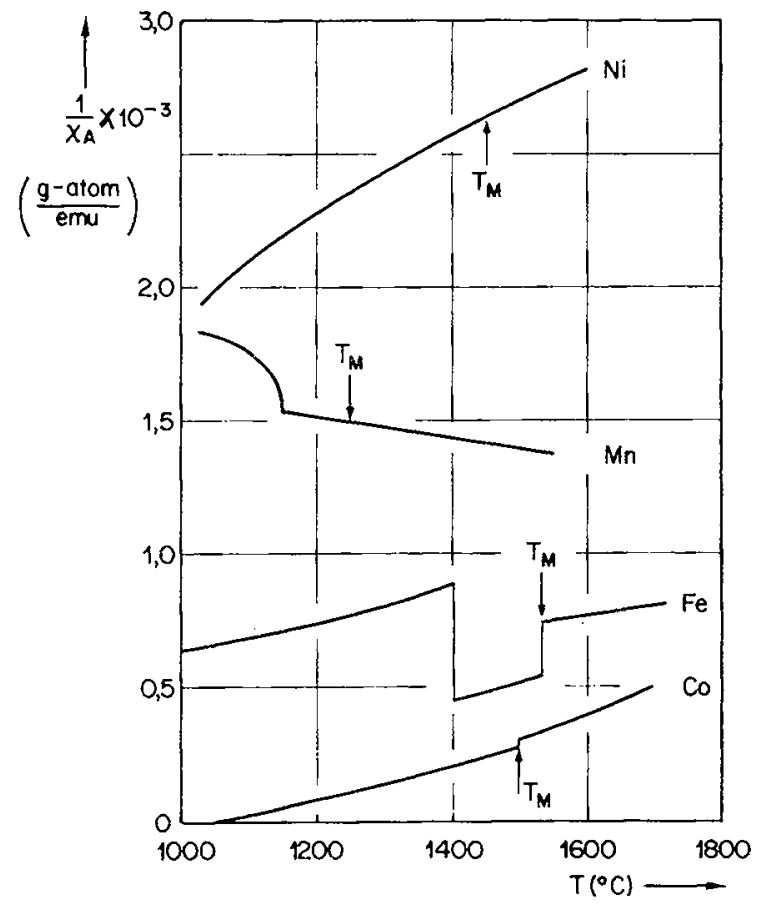

(a)

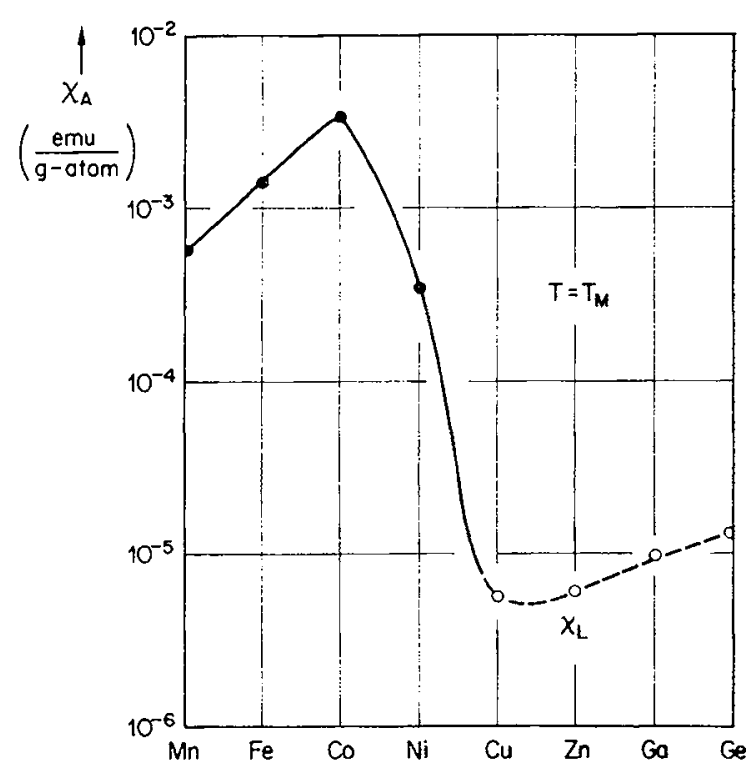

(b)

Fig. 4. - The magnetic susceptibility of liquid transition metals : a) reciprocal susceptibility as a function of temperature ; $b$ ) value of the susceptibility.

Figure 5 shows the magnetic susceptibility of liquid $\mathrm{Co}-$ and Fe-alloys on alloying. The susceptibility of Co-alloys decreases rapidly as a function of concentration of the normal metal. The same behavior has been observed for Ni-alloys. The susceptibility of Fe-alloys shows a maximum as a function of concentration of the normal metal and then decreases to the value of the susceptibility of the normal metal. A 


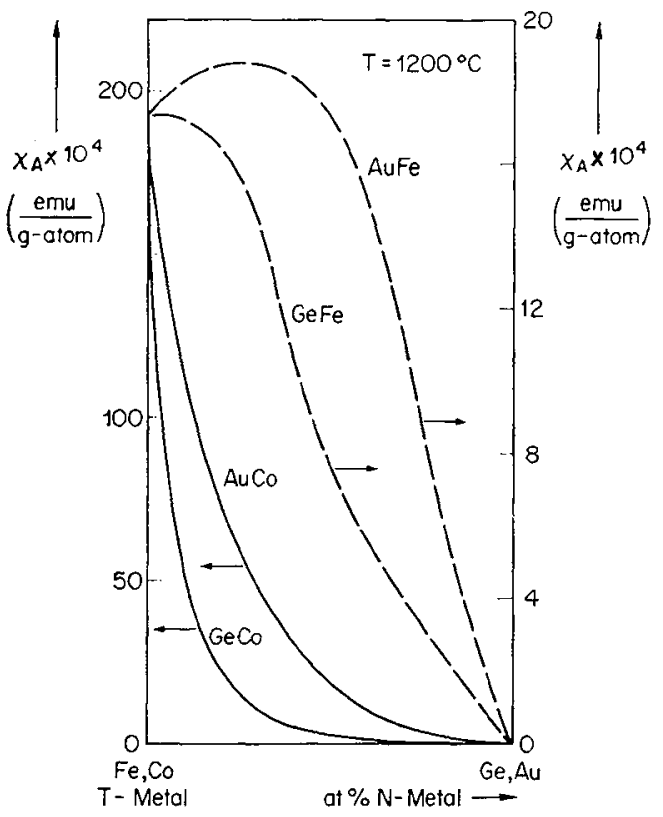

FIG. 5. - Experimental values of the magnetic susceptibility of liquid transition metal alloys.

similar maximum for the susceptibility as a function of concentration has been observed for $\mathrm{Mn}$-alloys.

The susceptibility values of transition metal alloys with $\mathrm{Cu}, \mathrm{Zn}$ and $\mathrm{Ga}$ show a systematic variation between the values of Au- and Ge-alloys.

The temperature coefficients of the reciprocal susceptibility as a function of concentration for liquid $\mathrm{Ni}$-alloys are shown in figure 6. For Ni-Au alloys the temperature coefficient is positive for almost all

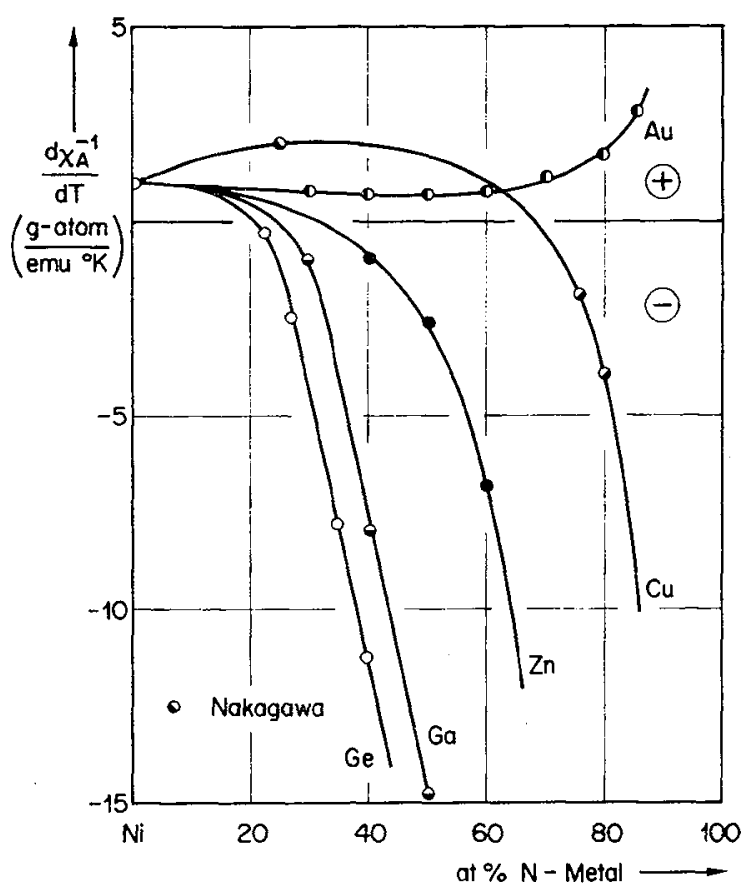

FrG. 6. - The temperature coefficient of the reciprocal susceptibility of liquid $\mathrm{Ni}$ alloys at their melting points. concentrations. All the other Ni-alloys show a change of the temperature coefficient. The concentration of the normal metal for which this change occurs increases from $\mathrm{Ge}$ to $\mathrm{Ga}$ and from $\mathrm{Zn}$ to $\mathrm{Cu}$ in the same manner as the Fermi energy decreases.

A general basis for the explanation of the variation of the susceptibility of paramagnetic alloys is given in a recent paper by Levin et al. [7]. This theory for the magnitude of the spin susceptibility of disordered binary alloys has been applied to the solid alloys Rh-Pd, Pt-Pd, Ni-Rh and Ni-Pd. Good quantitative agreement with experiment is obtained for the concentration dependence of the susceptibility in all these alloys. Calculations for the liquid alloys depend on a reasonable assumption for the density of states in the liquid state. Until such quantitative calculations are available, the experimental result of the magnetic susceptibility can be discussed quantitatively only for dilute alloys and qualitatively for the concentrated alloys.

The magnetic properties of dilute liquid alloys have been analyzed in terms of the Friedel-Anderson model [8]. For a detailed discussion see [1].

For concentrated alloys a similar behavior of the susceptibility and the temperature coefficient has been observed as in dilute alloys. Therefore the analysis of dilute alloys may be extended to more concentrated alloys. The susceptibility per mol for the alloy is given in terms of the enhanced spin susceptibility or of the weak scattering limit used by Levin et al. [7] by

$$
X_{\mathrm{A}}=c_{\mathrm{T}} \frac{N_{\mathrm{L}} B^{2} N_{\mathrm{d}}\left(E_{\mathrm{F}}\right) F(T)}{1-U_{\mathrm{eff}} N_{\mathrm{d}}\left(E_{\mathrm{F}}\right) F(T)}+c_{\mathrm{N}} X_{\mathrm{N}}
$$

with

$$
F(T)=1+\frac{\pi^{2}}{6} k_{\mathrm{B}}^{2} T^{2}\left[\frac{\partial^{2} \log N(E)}{\partial E^{2}}\right]_{E=E_{F}}
$$

where $c_{\mathrm{T}}$ and $c_{\mathrm{N}}$ are the concentrations of the transition and normal metal respectively. All the other symbols have the usual meaning. In a simple discussion the variation of the susceptibility and the temperature coefficient can be explained by the dependence of the density of states of the 3d-electrons $N_{\mathrm{d}}\left(E_{\mathrm{F}}\right)$ on concentration.

Any relevant discussion of the susceptibility has to be based on realistic density of states curves for alloys. An idea of the possible behavior of the density of states for liquid alloys of transition metals and monovalent noble metals can be deduced from the intensively investigated $\mathrm{Cu}-\mathrm{Ni}$ alloys in the solid state [9]. For liquid alloys of transition metals and polyvalent normal metals density of states which show typical differences from the first group can be proposed according to the susceptibility data.

In solid $\mathrm{Cu}-\mathrm{Ni}$ alloys the $\mathrm{Cu}$ d-states are located in the energy region associated with $\mathrm{Cu} d$-states in pure $\mathrm{Cu}$ and similarly $\mathrm{Ni}$ d-states are located in the energy region associated with $\mathrm{Ni}$ d-states in pure $\mathrm{Ni}$. 
Therefore, the Fermi energies as well as the positions of the d-bands relative to the Fermi energy are the same for pure $\mathrm{Cu}$ and $\mathrm{Ni}$ and $\mathrm{Cu}-\mathrm{Ni}$ alloys. In some way the $\mathrm{Cu}-\mathrm{Ni}$ alloys represent the simplest case of an alloy with fixed d-band and Fermi energy on alloying. A change of the center of the d-band as well as the Fermi energy is expected on alloying in other alloys, especially in alloys of transition metals with polyvalent normal metals [1].

The decrease and increase of the magnetic susceptibility as a function of concentration for Co or Ni-rich alloys on the one hand and for Fe-rich alloys on the other hand is governed by the decrease or increase of the density of states at the Fermi energy in $\mathrm{Co}-$ or $\mathrm{Ni}-$ and $\mathrm{Fe}$-alloys, respectively.

The temperature coefficient of the susceptibility may also be explained in terms of the density of states at the Fermi energy. According to eq. (6) a positive temperature coefficient of the reciprocal susceptibility is expected for a maximum and a negative temperature coefficient for a minimum of the density of states at the Fermi energy. The change of sign of the temperature coefficient as a function of concentration occurs at the transition region.

\section{Liquid rare-earth metals and their alloys.}

3.1 HaLl COEFFICIENTS. - Figure 7 shows the Hall coefficients of $\mathrm{Ce}, \mathrm{La}$ and $\mathrm{Cd}$ close to their melting points. The data of the Hall coefficient of $\mathrm{Cd}$ are given for comparison. In contrast to liquid normal metals a positive Hall coefficient has been observed for liquid $\mathrm{Ce}$ and $\mathrm{La}$. These Hall coefficients are independent of temperature in the liquid state. The Hall coefficient of Ce does not change at the melting point. A change has been observed at the temperature $T_{\mathbf{p}}$ of the phase transition from the fcc to the bcc phase. The Hall coefficient of $\mathrm{La}$ changes at both the temperature of the phase transition and at the melting point.
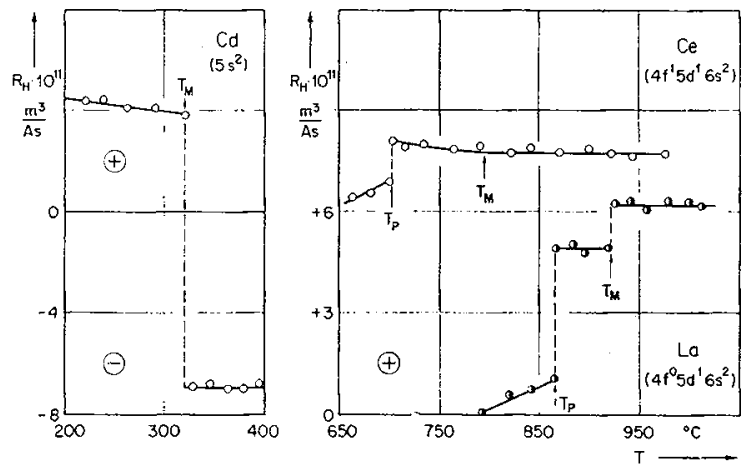

FrG. 7. - The Hall coefficients of liquid $\mathrm{Cd}, \mathrm{Ce}$ and $\mathrm{La}$ as a function of temperature.

The Hall coefficients of the light rare-earth metals, $\mathrm{La}, \mathrm{Ce}, \mathrm{Pr}, \mathrm{Nd}$ increase with increasing number of the $4 \mathrm{f}$-electrons. At present there are no other explanations of the positive Hall coefficients in liquid rare- earths than those given for liquid transition metals. In the solid state the Hall coefficients of rare earths were discussed by assuming holes [10]. This is surprising in view of the Hall coefficient of $\mathrm{Ce}$ which does not change at the melting point.

The behavior of the Hall coefficient of $\mathrm{Ce}$ on alloying with $\mathrm{Cu}$ is shown in figure 8. The Hall coefficient is negative for pure liquid $\mathrm{Cu}$, passes through zero at 20 at. $\% \mathrm{Ce}$ and is positive for pure liquid $\mathrm{Ce}$. A linear variation of the Hall coefficient with $\mathrm{Ce}$ concentration up to 40 at.- $\% \mathrm{Ce}$ has been observed. Beyond this concentration it remains constant at the value of pure liquid $\mathrm{Ce}$.

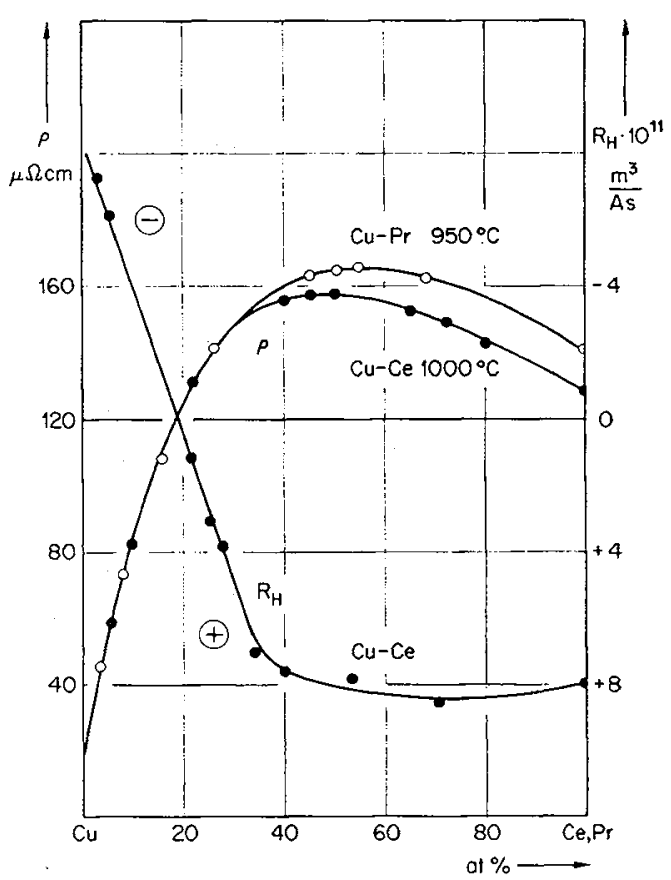

FIG. 8. - The Hall coefficient and the electrical resistivity of liquid $\mathrm{Cu}-\mathrm{Ce}$ and $\mathrm{Cu}-\mathrm{Pr}$ alloys.

3.2 Electrical Resistivity. - Table I gives a survey of the electrical resistivity at the melting point of liquid rare earth metals. The resistivity values of the investigated light rare earth metals do not vary much from one to another. The values are of the same magnitude as for $\mathrm{Bi}$ and $\mathrm{Fe}$. Therefore an explanation of the electrical resistivity in terms of the resonance scattering formula has been suggested [11] only by taking into account the 5d-states rather than the 4 f-states.

\section{TABLE I}

The electrical resistivity of liquid rare earth metals at the melting point in $\mu \Omega \mathrm{cm}$

$\begin{array}{cccccc}\mathrm{La} & \mathrm{Ce} & \mathrm{Pr} & \mathrm{Nd} & \mathrm{Gd} & \mathrm{Tb} \\ \overline{135} & \overline{127} & \overline{138} & \overline{155} & \overline{195} & \frac{-}{190}\end{array}$


Recent XPS measurements [12], calculations of an energy scheme in the renormalized atom procedure [13] on solid rare earth metals and calculations of the density of states of liquid rare earths by the cluster method [14] can be interpreted as showing a rather high density of states at the Fermi energy due to the 5 d electrons and a peak with increasing difference between its center and the Fermi energy due to the $4 \mathrm{f}$-electrons. In other words, the difference between the $4 \mathrm{f}$-states and the Fermi energy increases from $\mathrm{Ce}$ to $\mathrm{Nd}$, whereas the difference between the resonance energy of the $5 \mathrm{~d}$-states and the Fermi energy is more or less equal for the different light rare earth investigated so far. Thus, if the resistivity were due to resonance scattering by the $4 \mathrm{f}$-states it would decrease from $\mathrm{Ce}$ to $\mathrm{Nd}$ in contradiction to the measurements.

For the heavy rare earth metals $\mathrm{Gd}$ and $\mathrm{Tb}$ (see also Fig. 9) [15] large values of the electrical resistivity have been observed. Such values of about $190 \mu \Omega \mathrm{cm}$ are the largest found for pure liquid metals together with the values of about $300 \mu \Omega \mathrm{cm}$ for pure liquid $\mathrm{Ba}$ [15] and $\mathrm{Eu}$ [15]. At present there are no explanations for the values of the resistivity for $\mathrm{Tb}$ and $\mathrm{Gd}$. For liquid $\mathrm{Ba}$ a theoretical value has been suggested by taking into account d-resonance scattering [2]. More recently the idea of a p-resonance and s-scattering has been discussed due to the calculations of the phase shifts of $\mathrm{Ba}[14]$.

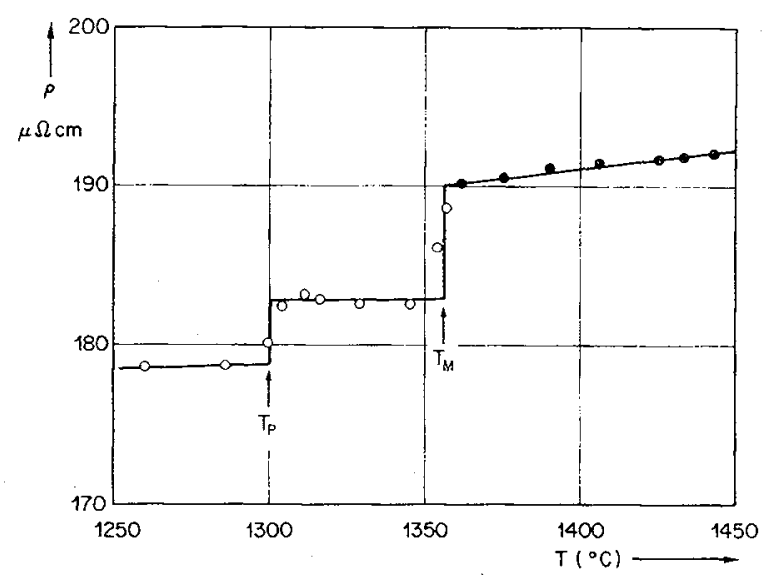

FIG. 9. - The electrical resistivity of Terbium.

The variation of the electrical resistivity on alloying is shown in figure 8 . The electrical resistivity for liquid alloys of rare-earth metals $\mathrm{Ce}$ and $\mathrm{Pr}$ with $\mathrm{Cu}$ shows a pronounced maximum. The value of this maximum is eight times the resistivity for pure liquid $\mathrm{Cu}$. The resistivity values coincide for the $\mathrm{Cu}$-rich side and the curves separate on the rare-earth metalrich side due to the slightly different values of pure liquid $\mathrm{Ce}$ and $\mathrm{Pr}$.

3.3 MAGNetic SUSCEPTiBILITY. - The magnetic susceptibility of pure Ce, $\mathrm{Pr}$ and Nd follows a CurieWeiss law up to room temperature. The effective magnetic moment corresponds to one localized 4felectron for $\mathrm{Ce}$, two for $\mathrm{Pr}$ and three for $\mathrm{Nd}$. At higher temperatures and in the liquid state, a deviation to larger values of the magnetic susceptibility occurs. La has no 4f-electron and shows the usual temperature independent Pauli paramagnetism, enhanced by a factor of 10 compared with the Pauli-Landau susceptibility of liquid normal metals.

The magnetic susceptibility of the liquid alloys of $\mathrm{Ce}$ with $\mathrm{Cu}, \mathrm{Sn}, \mathrm{La}$ and $\operatorname{Pr}$ (Fig. 10) varies nearly linearly with concentration and $\mathrm{Ce}$ retains its local magnetic moment in such alloys.

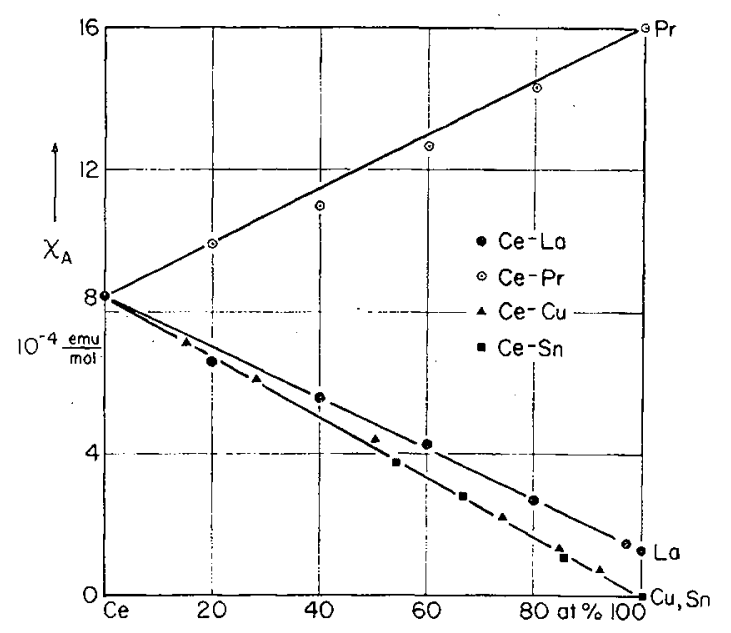

Fro. 10. - The magnetic susceptibility of liquid alloys of $\mathrm{Ce}$ with $\mathrm{Cu}, \mathrm{Sn}, \mathrm{La}$ and $\mathrm{Pr}$.

The most interesting features of the magnetic susceptibility of liquid rare-earth metals on alloying have been observed in $\mathrm{Co}$-Ce alloys.

Figure 11 shows the magnetic susceptibility and the temperature coefficient of the reciprocal susceptibility of liquid $\mathrm{Co}-\mathrm{Ce}$ alloys. The magnetic susceptibility of liquid $\mathrm{Ce}$ decreases on alloying with $\mathrm{Co}$, passes through a minimum and then tends to the large value of liquid $\mathrm{Co}$. The temperature coefficient of the reciprocal susceptibility remains nearly constant for $\mathrm{Ce}-$ rich alloys, goes to zero at 62 at.- $\% \mathrm{Ce}$, and becomes negative. Also for Co-rich alloys the temperature coefficient decreases to zero at 40 at. $\% \mathrm{Ce}$ and becomes negative with decreasing Co-concentration.

The observed magnetic susceptibility and the disappearance of the magnetic moment of $\mathrm{Ce}$ in liquid $\mathrm{Ce}-\mathrm{Co}$ alloys can be explained by a change of the occupation number of the $4 \mathrm{f}-\mathrm{level}$ of $\mathrm{Ce}$, as assumed in solid $\mathrm{Ce}$ under pressure.

So far, solid $\mathrm{Ce}$ has been characterized in the $\beta$ and $\gamma$-phases by the presence of a narrow 4f-level close to the Fermi energy [16] and in these phases Ce exhibits a localized magnetic moment. But under pressure Ce looses its localized magnetic moment in the $\alpha$-phase [17] and becomes even superconducting in the $\alpha^{\prime}$-phase [18]. The disappearance of the localized magnetic moment at the $\alpha-\gamma$ transition has been explain- 

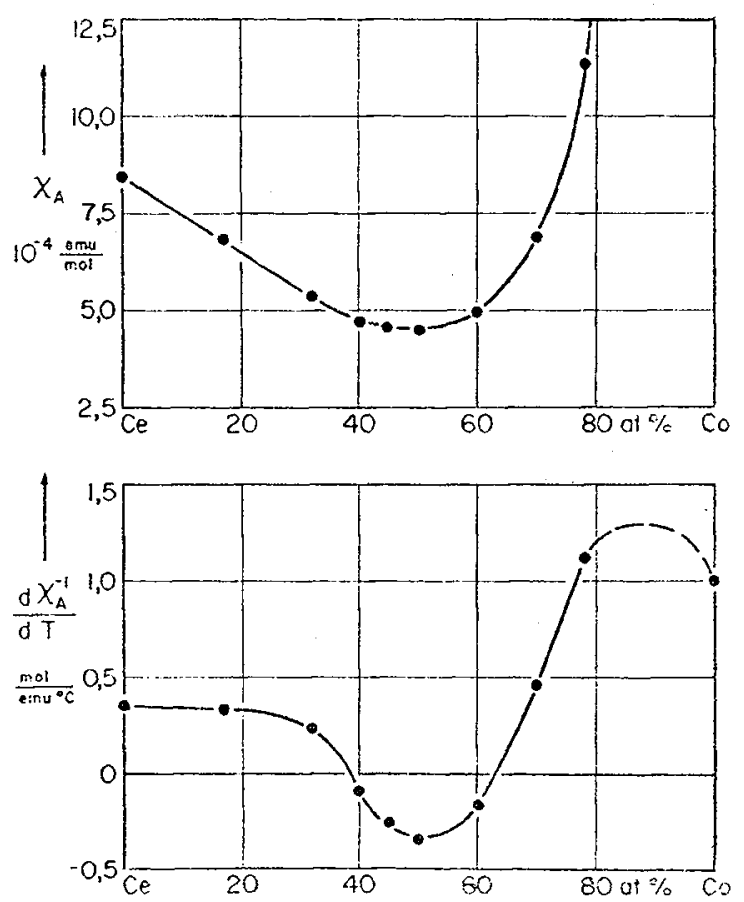

FIG. 11. - The magnetic susceptibility and the temperature coefficient of the reciprocal susceptibility of liquid Co-Ce alloys.

ed by the change of the occupation number of the 4f-level $[16,19]$.

In liquid $\mathrm{Co}-\mathrm{Ce}$ alloys a similar change of the occupation number due to a shift of the Fermi energy relative to the $4 \mathrm{f}$-level has been expected.

Figure 12 shows the density of states of $\mathrm{Ce}, \mathrm{Co}$ and $\mathrm{Co}-\mathrm{Ce}$ alloys obtained by a cluster method [14]. Such a method gives reasonable values for the $E_{\mathrm{MTZ}}$ (the bottom of the bands), the Fermi energy $E_{F}$ and the position of the $4 \mathrm{f}$-states. Thus we have a first verification of the so far suggested density of states for $\mathrm{Co}-\mathrm{Ce}$ alloys. It follows very clearly from the calculated density of states that at 60 at. $\%$ Ce in Co the Fermi energy coincides with the 4f-level and even for smaller Ce-concentrations the 4f-level lies above the Fermi energy, if $4 \mathrm{f}^{1}$ were the occupation.

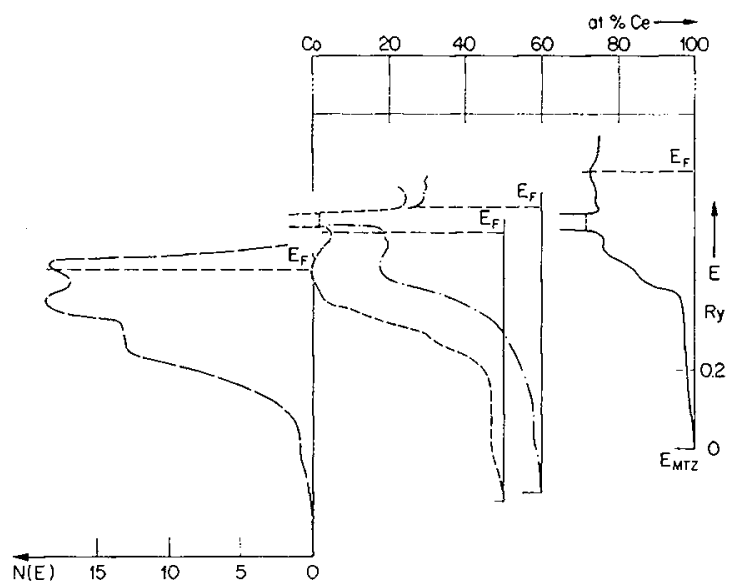

Fig. 12. - The density of states of liquid $\mathrm{Co}, \mathrm{Ce}$ and $\mathrm{Co}-\mathrm{Ce}$ alloys.
Preliminary results of the electrical resistivity and the Hall coefficient [20] of liquid $\mathrm{Co}-\mathrm{Ce}$ show, that the electrical resistivity of $\mathrm{Ce}$ increases to a maximum on alloying with Co and that the positive Hall coefficients of $\mathrm{Ce}$ decrease to zero and even to negative values on alloying with Co. In the range of 40 to 60 at. $\%$ Ce the alloys tend to be more free-electron like.

The magnetic susceptibility and the temperature coefficient of the reciprocal susceptibility of liquid Co-La alloys are shown in figure 13. The susceptibility of liquid La-rich alloys remains nearly constant on alloying with $\mathrm{Co}$, increases slightly for Co-concentrations higher than 20 at.- $\% \mathrm{Co}$ and tends to the large value of liquid $\mathrm{Co}$. The temperature coefficient of the reciprocal susceptibility shows a S-shaped behavior. The temperature coefficient of pure liquid $\mathrm{La}$ is zero, becomes negative on alloying with $\mathrm{Co}$ and passes through a minimum. For increasing Co concentrations the temperature coefficient shows a maximum and decreases to the value of pure liquid Co.

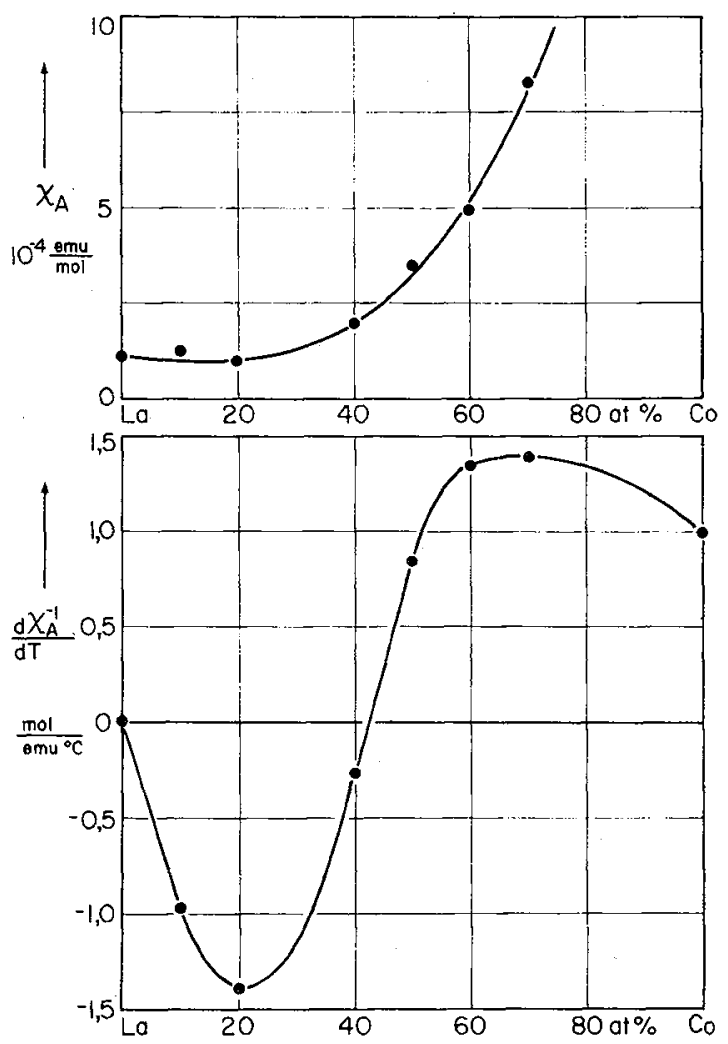

FIG. 13. - The magnetic susceptibility and the temperature coefficient of the reciprocal susceptibility of liquid Co-La alloys.

Figure 14 shows the magnetic susceptibility and the temperature coefficient of the reciprocal susceptibility of liquid Co-Pr alloys. A minimum of the magnetic susceptibility near 50 at.- $\% \operatorname{Pr}$ has been observed. The temperature coefficient of the reciprocal susceptibility is always positive and increases from $\mathrm{Pr}$ to Co. The localized 4f-electrons of liquid Pr do not disappear due to the larger difference between the Fermi energy and the 4f-level of liquid Pr. 

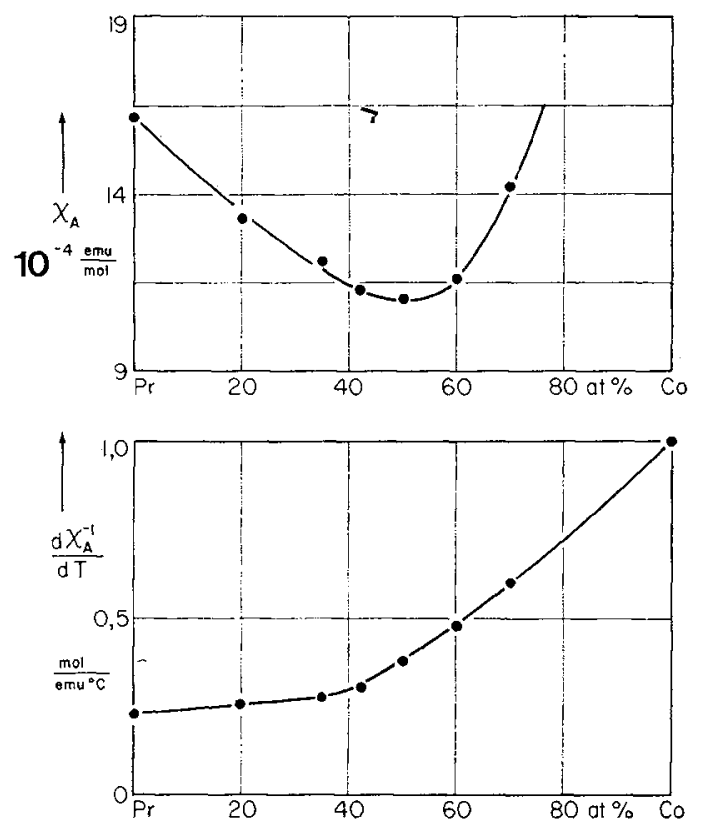

FIG. 14. - The magnetic susceptibility and the temperature coefficient of the reciprocal susceptibility of liquid Co-Pr alloys.
4. Conclusion. - The reviewed experimental data on liquid transition and rare-earth metals and first of all their alloys may be helpful for a better understanding of these metals in general. Isotropy and miscibility of the liquid state allow more systematic investigations than in the solid state, which greatly help in finding theoretical explanations.

Acknowledgment. - We would like to express our sincere gratitude to dipl. phys. ETH Annamarie Zimmermann for her cooperation, stimulating discussions and suggestions during the last three years in the "Liquid Metals " group.

The authors are very much indebted to Prof. W. Baltensperger, Prof. K. H. Bennemann, Prof. N. F. Mott, Prof. J. M. Ziman, Dr. R. Evans and Dr. A. ten Bosch for stimulating discussions and suggestions. The excellent experimental measurements by E. Hauser and R. Müller are gratefully acknowledged.

Finally, we would like to thank the « Schweizerische Nationalfonds zur Förderung der wissenschaftlichen Forschung » and the Research Center of Alusuisse for financial support.

\section{References}

[1] Busch, G. and Guentherodt, H.-J., Solid State Phys., to be published.

[2] Evans, R., Greenwood, D. A. and Lloyd, P., Phys. Lett. 35A (1971) 57.

Evans, R., GyoRfFy, B. L., SZABo, N. and ZiMAN, J. M., Proc. 2nd Intern. Conf. Liq. Metals, Tokyo, p. 319, 1973.

[3] Evans, R., Guentherodt, H.-J., Kuenzi, H. U. and Zimmermann, A., Phys. Lett. 38A (1972) 151.

[4] Dreirach, O., Evans, R., GuentherodT, H.-J. and Kuenzi, H. U., J. Phys. F (Metal Phys.) 2 (1972) 709.

[5] RuppersBerg, H. and SEEmANN, H.-J., $Z$. Naturforsch. 21a (1966) 820.

Waseda, Y., Suzuki, K., Tamaki, S. and Takeuchu, S., Phys. Stat. Sol, 39 (1970) 181.

WaSeda, Y. and SuzukI, K., Phys. Stat. Sol. 39 (1970) 669.

[6] Nakagawa, Y., J. Phys. Soc. Japan 11 (1956) 855.

KOHLHAAS, R., Archiv f. Eisenhüttenwesen 36 (1965) 437.

KohlmaAs, R. and Weiss, W. D., Z. Naturforsch 24a (1969) 287.

Urbain, G. and Uebelacker, E., Adv. Phys. 16 (1967) 429.

[7] Levin, K., Bass, R. and Bennemann, K. H., Phys. Rev. B 6 (1972) 1865.

[8] Friedel, J., Nuovo Cimento 2 (1958) 287.

Anderson, P. W., Phys. Rev. 124 (1961) 41.
[9] SeIr, D. H. and SpICer, W. E., Phys. Rev. B 1 (1970) 1676. Clift, J., Curry, C. and Thompson, B. J., Phil. Mag. 8 (1963) 593.

Azaroff, L. V. and Das, B. N., Phys. Rev. 134 (1964) A 747.

Huefner, S., Wertheim, G. K., Cohen, R. L. and WerNICK, J. H., Phys. Rev. Lett. 28 (1972) 488.

Stocks, G. M., Williams, R. W. and Taulkner, J. S., Phys. Rev. B 4 (1971) 4390.

[10] Gschneidner, A., Jr. and Smoluchowski, J., J. LessCommon Metals 5 (1963) 374.

[11] Guentherodt, H.-J. and Zimmermann, A., Phys. kondens. Mat. 1973.

[12] Baer, Y. and Busch, G., Phys. Rev, Lett. 31 (1973) 35.

[13] Herbst, J. F., Lowy, D. N. and WATson, R. E., Phys. Rev. B 6 (1972) 1913.

[14] Keller, J., J. Physique 35 (1974) 379.

[15] HAUSER, E, diploma thesis ETH 1973, to be published.

[16] CoQblin, B., J. Physique 32 (1971) C1-599.

[17] Macpherson, M. R., Everett, G. E., Wohlleben, D. and MAPle, M. B., Phys. Rev. Lett. 26 (1971) 20.

[18] Witrig, J., Phys. Rev. Lett. 21 (1968) 1250.

[19] Ramirez, R. and Falicov, L. M., Phys. Rev. B 3 (1971) 2425.

[20] Busch, G., Guenterhodt, H.-J., Kuenzi, H. U. and MUeller, R., to be published. 